\title{
The isometries of the cut, metric and hypermetric cones
}

\author{
Antoine Deza • Boris Goldengorin • \\ Dmitrii V. Pasechnik
}

Received: June 17, 2003 / Revised: August 2, 2005 / Accepted: August 11, 2005

(C) Springer Science + Business Media, Inc. 2006

\begin{abstract}
We show that the symmetry groups of the cut cone $\mathrm{Cut}_{n}$ and the metric cone $\operatorname{Met}_{n}$ both consist of the isometries induced by the permutations on $\{1, \ldots, n\}$; that is, $I s\left(\mathrm{Cut}_{n}\right)=I s\left(\mathrm{Met}_{n}\right) \simeq \operatorname{Sym}(n)$ for $n \geq 5$. For $n=4$ we have $I s\left(\mathrm{Cut}_{4}\right)=I s\left(\mathrm{Met}_{4}\right) \simeq$ $\operatorname{Sym}(3) \times \operatorname{Sym}(4)$. This result can be extended to cones containing the cuts as extreme rays and for which the triangle inequalities are facet-inducing. For instance, $\operatorname{Is}\left(\operatorname{Hyp}_{n}\right) \simeq \operatorname{Sym}(n)$ for $n \geq 5$, where $\mathrm{Hyp}_{n}$ denotes the hypermetric cone.
\end{abstract}

Keywords Polyhedral combinatorics $\cdot$ Metric cone $\cdot$ Hypermetric cone $\cdot$ Symmetry group

\section{Introduction and notation}

The $\left(\begin{array}{l}n \\ 2\end{array}\right)$-dimensional cut cone $\mathrm{Cut}_{n}$ is usually introduced as the conic hull of the incidence vectors of all the cuts of the complete graph on $n$ nodes. More precisely, given a subset $S$ of $V_{n}=\{1, \ldots, n\}$, the $c u t$ determined by $S$ consists of the pairs $(i, j)$ of elements of $V_{n}$ such that exactly one of $i, j$ is in $S$. By $\delta(S)$ we denote both the cut and its incidence vector

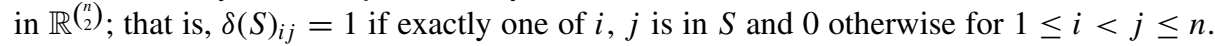
By abuse of notation, we use the term cut for both the cut itself and its incidence vector, so

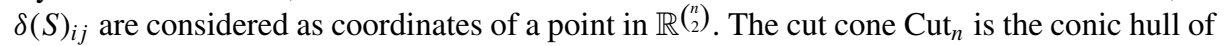

\footnotetext{
A. Deza

Dept. of Computing and Software, McMaster University, Hamilton, Ontario, Canada L8S 4K1

e-mail: deza@mcmaster.ca; Web:/http://www.cas.mcmaster.ca/ deza/

B. Goldengorin

Dept. of Econometrics and Operations Research, University of Groningen, P.O. Box 800, 9700 AV

Groningen, The Netherlands and Dept. of Applied Mathematics, Khmelnitsky National University,

Ukraine

e-mail: b.goldengorin@rug.nl

D. V. Pasechnik $(\square)$

School of Physical and Mathematical Sciences, Nanyang Technological University, 50 Nanyang Avenue, Singapore 639798

e-mail: dima@ntu.edu.sg; Web:/http://www.ntu.edu.sg/home/dima/
} 
all $2^{n-1}-1$ nonzero cuts, and the cut polytope cut $_{n}$ is the convex hull of all $2^{n-1}$ cuts. The cut cone and one of its relaxation - the metric cone $\mathrm{Met}_{n}$ - can also be defined in terms of finite metric spaces in the following way. For all 3 -sets $\{i, j, k\} \subset\{1, \ldots, n\}$, we consider the following inequalities.

$$
\begin{aligned}
& x_{i j}-x_{i k}-x_{j k} \leq 0, \\
& x_{i j}+x_{i k}+x_{j k} \leq 2 .
\end{aligned}
$$

(1) induce the $3\left(\begin{array}{l}n \\ 3\end{array}\right)$ facets which define the metric cone $\mathrm{Met}_{n}$. Then, bounding the latter by the $\left(\begin{array}{l}n \\ 3\end{array}\right)$ facets induced by (2) we obtain the metric polytope met $_{n}$. The facets defined by (1) (resp. by (2)) can be seen as triangle (resp. perimeter) inequalities for distance $x_{i j}$ on $\{1, \ldots, n\}$. While the cut cone is the conic hull of all, up to a constant multiple, $\{0,1\}$-valued extreme rays of the metric cone, the cut polytope cut $_{n}$ is the convex hull of all $\{0,1\}$-valued vertices of the metric polytope. The link with finite metric spaces is the following: there is a natural $1-1$ correspondence between the elements of the metric cone and all the semi-metrics on $n$ points, and the elements of the cut cone correspond precisely to the semi-metrics on $n$ points that are isometrically embeddable into some $l_{1}^{m}$, see [1]. It is easy to check that such minimal $m$ is smaller than or equal to $\left(\begin{array}{c}n \\ 2\end{array}\right)$.

One of the motivations for the study of these polyhedra comes from their applications in combinatorial optimization, the most important being the MAXCUT and multicommodity flow problems. For instance, the linear programming approach to MAXCUT involves considering cutting planes that are needed to be added to $\operatorname{Met}_{n}$ to obtain $\mathrm{Cut}_{n}$. These cutting planes define cones $C_{n}$ such that $\mathrm{Cut}_{n} \subseteq C_{n} \subseteq \operatorname{Met}_{n}$. Perhaps the most well-known example of such a $C_{n}$ is the hypermetric cone $\mathrm{Hyp}_{n}$ which is defined by facets induced by inequalities generalizing the triangle inequalities. For a detailed study of those polyhedra and their applications in combinatorial optimization we refer to DEZA and LAURENT [9].

\section{Main result}

One important feature of the metric and cut polyhedra is their very large symmetry group. We recall that the symmetry group $I s(P)$ of a polyhedron $P$ is the group of isometries preserving $P$ and that an isometry is a linear transformation preserving the Euclidean distance. While the symmetry groups of the cut and metric polytopes are known, the question whether the cut and metric cones admit no other isometry than the ones induced by $\operatorname{Sym}(n)$ was open, see [7, 8, 14]. More precisely, for $n \geq 5, I s\left(\operatorname{met}_{n}\right)=I s\left(\mathrm{cut}_{n}\right)$ and both are induced by permutations on $V_{n}=\{1, \ldots, n\}$ and switching reflections by a cut and, for $n \geq 5$, we have $\left|I s\left(\operatorname{met}_{n}\right)\right|=2^{n-1} n$ !, see [8]. Given a cut $\delta(S)$, the switching reflection $r_{\delta(S)}$ is defined by $y=r_{\delta(S)}(x)$ where $y_{i j}=1-x_{i j}$ if $(i, j) \in \delta(S)$ and $y_{i j}=x_{i j}$ otherwise.

The aim of this article is to show that $I s\left(\mathrm{Cut}_{n}\right)=I s\left(\mathrm{Met}_{n}\right) \simeq \operatorname{Sym}(n)$ for $n \geq 5$ and $I s\left(\mathrm{Cut}_{4}\right)=I s\left(\mathrm{Met}_{4}\right) \simeq \operatorname{Sym}(3) \times \operatorname{Sym}(4)$. A part of Theorem (1) was conjectured in [7] and was substantiated by computer calculations of the automorphism group of the ridge graph of $\operatorname{Met}_{n}$ for $n \leq 20$. We recall that ridge graph of a polyhedra $C_{n}$ is the graph which vertices are the facets of $C_{n}$, two facets being adjacent if and only if their intersection is a face of codimension 2 of $C_{n}$. In other words, the ridge graph of $C_{n}$ is the skeleton of the dual of $C_{n}$. 
Theorem 1. The symmetry groups of the cones $\mathrm{Met}_{n}$ and $\mathrm{Cut}_{n}$ are isomorphic to $\operatorname{Sym}(n)$ for $n \geq 5$ and to $\operatorname{Sym}(3) \times \operatorname{Sym}(4)$ for $n=4$.

The proof of Theorem 1 is given in Section 3. In Section 3.1 we characterize $I s\left(\mathrm{Met}_{n}\right)$, in Section 3.2 we show that $I s\left(\mathrm{Cut}_{n}\right)=I s\left(\mathrm{Met}_{n}\right)$ and in Section 3.3 we generalize Theorem 1 in the following way.

Theorem 2. Let $C_{n}$ be a cone satisfying

(i) the cuts are extreme rays of $C_{n}$,

(ii) the triangle inequalities are facet-inducing for $C_{n}$.

Then any isometry of $C_{n}$ is induced by a permutation on $\{1, \ldots, n\}$.

A cone $C_{n}$ satisfying the condition (i) and (ii) of Theorem 2 is cone satisfying $\mathrm{Cut}_{n} \subseteq C_{n} \subseteq$ $\mathrm{Met}_{n}$. Apart from $\mathrm{Met}_{n}$ and $\mathrm{Cut}_{n}$ themselves, a well-known example of such a cone $C_{n}$ is the hypermetric cone $\mathrm{Hyp}_{n}$ defined by the following hypermetric inequalities (3) which generalize the triangle inequalities:

$$
\sum_{1 \leq i<j \leq n} b_{i} b_{j} x_{i j} \leq 0 \quad \text { with } \sum_{i=1}^{n} b_{i}=1
$$

We recall that $I s\left(\operatorname{Hyp}_{n}\right)$ contains the isometries induced by the permutations on $\{1, \ldots, n\}$.

Corollary 1. The symmetry group of $\mathrm{Hyp}_{n}$ is isomorphic to $\operatorname{Sym}(n)$ for $n \geq 5$ and to $\operatorname{Sym}(3) \times \operatorname{Sym}(4)$ for $n=4$.

\section{Proofs}

We first prove Theorem 1 for $\mathrm{Met}_{n}$ by showing that its ridge graph $G_{n}$ for $n>4$ has the automorphism group $\operatorname{Sym}(n)$; the symmetry group of $\mathrm{Met}_{4}$ is constructed directly. We complete the proof of Theorem 1 by showing that $G_{n}$ is an induced subgraph of the ridge graph of $\mathrm{Cut}_{n}$ that is invariant under the isometries of $\mathrm{Cut}_{n}$. Finally, we prove Theorem 2 by noticing that $\mathrm{Cut}_{n}$ can be replaced by any cone $C_{n}$ satisfying $\mathrm{Cut}_{n} \subseteq C_{n} \subseteq \mathrm{Met}_{n}$. The group-theoretic notation used in the paper can be found e.g. in [3].

\subsection{The group $I s\left(\mathrm{Met}_{n}\right)$ for $n \geq 5$}

\subsubsection{Is $\left(\mathrm{Met}_{n}\right)$ for $n \geq 4$}

Note that the isometries act faithfully on the facets; that is, the only isometry that stabilizes each facet of $\mathrm{Met}_{n}$ is the trivial one. As each permutation on $V_{n}$ is an isometry, in order to prove the statement for $n \geq 5$, it suffices to show that the automorphism group $A=\operatorname{Aut}\left(G_{n}\right)$ of the ridge graph $G_{n}$ is isomorphic to $\operatorname{Sym}(n)$.

The facets of $\operatorname{Met}_{n}$ naturally correspond to $\{0,1,-1\}$ vectors of length $\left(\begin{array}{l}n \\ 2\end{array}\right)$ with one positive and two negative entries. Two triangle facets $u$ and $v$ are adjacent in $G_{n}$, i.e. intersect on a face of codimension 2, if and only if they are non-conflicting; that is, there is no position $i j$ such that the corresponding entries $u_{i j}$ and $v_{i j}$ are nonzero and of opposite sign, see [5,9]. 


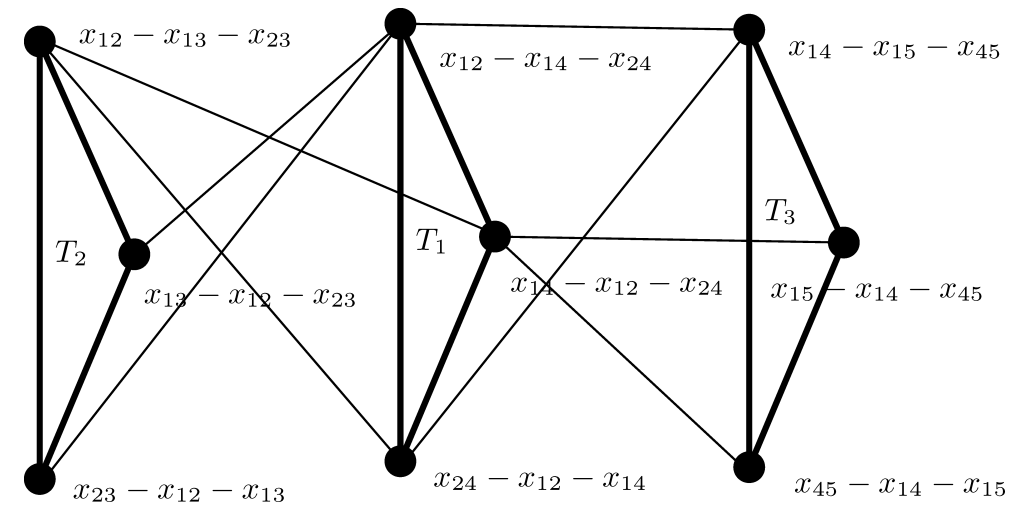

Fig. 1 Adjacencies between Triangles $T_{1}=\{1,2,4\}, T_{2}=\{1,2,3\}$, and $T_{3}=\{1,4,5\}$

As already observed in [5], instead of working with $G_{n}$, it appears to be easier to work with its complement $\bar{G}_{n}$ which has the same automorphism group. Observe that if two vertices $u$ and $v$ are conflicting then they have either exactly one nonzero entry in common, or all the three nonzero entries in common. Indeed, if $u_{i k} u_{j k} u_{i j} v_{i j} \neq$ 0 and $v_{i k^{\prime}} v_{j k^{\prime}} \neq 0$, then either $k=k^{\prime}$, and the latter holds, or $k \neq k^{\prime}$, and the former holds.

The subgraph induced on the neighbours of a vertex $u$ of $\bar{G}_{n}$ is isomorphic to the disjoint union of $n-3$ hexagons on a common edge $\left(u^{\prime}, u^{\prime \prime}\right)$, see [5]. It is easy to see that $u, u^{\prime}$, and $u^{\prime \prime}$ all have the same zero entries. From now on let us refer to such a type of 3-clique in $\bar{G}_{n}$ as a Triangle. The Triangles $\left\{u, u^{\prime}, u^{\prime \prime}\right\}$ form an orbit of $A$, as the number of common neighbours of an edge of Triangle is $n-2$, bigger than for the edges of another types, where it is just 2.

Let us look at the edges between any two given Triangles $T_{1}$ and $T_{2}$. If they do not share a common nonzero entry then there are no edges in between. Otherwise there are exactly 4 edges (see Fig. 1), and, ignoring the edges of $T_{1}$ and $T_{2}$, the subgraph induced on their 6 vertices is the disjoint union of two 2-paths. This implies that every $g \in A$ stabilizing $T_{1}$ and $T_{2}$ either fixes $T_{1}$ pointwise, or induces an element of order 2 on the vertices of $T_{1}$. Let $T_{3}$ be a third Triangle having a common entry with $T_{1}$, the different one than the common entry of $T_{1}$ and $T_{3}$. Then the 2-path between $T_{1}$ and $T_{3}$ with the middle point in $T_{1}$ does not intersect the 2-path between $T_{1}$ and $T_{2}$ with the middle point in $T_{1}$. Hence every $g \in A$ stabilizing $T_{1}, T_{2}$ and $T_{3}$ fixes $T_{1}$ pointwise. Therefore if $g \in A$ stabilizes all the Triangles, then $g$ is the identity; that is, $A$ acts faithfully on the set of Triangles.

Let us define the graph $\Gamma_{n}$ on the Triangles, two Triangles are adjacent if there is an edge of $\bar{G}_{n}$ joining a vertex of the first Triangle with a vertex of the second Triangle (so there are 4 edges forming the disjoint union of 2-paths that join them). To complete the proof, it suffices to show that $\operatorname{Aut}\left(\Gamma_{n}\right) \cong A$. The latter is in fact not true for $n=6$, and we shall treat this case separately.

Note that $\Gamma_{n}$ is naturally isomorphic to the first subconstituent of the Johnson scheme $J(n, 3)$, in other words, the graph with the vertex set $\left(\begin{array}{c}V_{n} \\ 3\end{array}\right)$, two vertices adjacent if the corresponding 3-subsets intersect in a 2-subset. Automorphism groups of these graphs are described e.g. in [10, 12], and were known at least since [13]. We give here a self-contained treatment, as the particular case we are dealing with is a simple one. Note that $\Gamma_{5}$ is the 


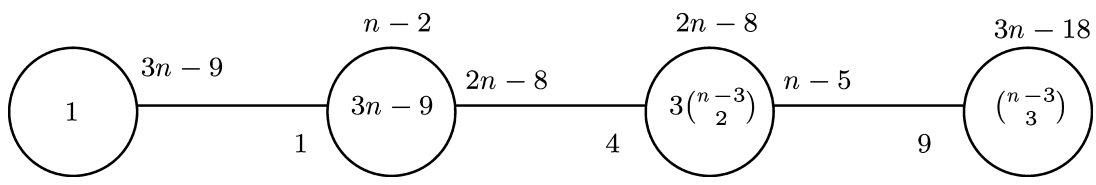

Fig. 2 The distribution diagram of $\Gamma_{n}$

complement of the Petersen graph, and it is well-known that its automorphism group is $\operatorname{Sym}(5)$. This completes the proof for $n=5$.

For $n>5$, the graph $\Gamma_{n}$ is distance-regular of diameter 3, see e.g. [2] for this notion.

The subgraph $\Omega$ induced on the neighbourhood $\Gamma_{n}(v)$ of a vertex $v$ is isomorphic to the line graph of $K_{3, n-3}$.

If $n>6$ then the automorphism group of $\Omega$ is isomorphic to $\operatorname{Sym}(3) \times \operatorname{Sym}(n-3)$. It suffices to show that the stabilizer of $v$ in $\operatorname{Aut}\left(\Gamma_{n}\right)$ acts faithfully on $\Gamma_{n}(v)$, as then it will coincide with the one in $\operatorname{Aut}\left(\Gamma_{n}\right) \cap \operatorname{Sym}(n)$. Let $H$ be the kernel of the latter action. As any vertex at distance 2 from $v$ is adjacent to exactly 4 vertices in $\Gamma_{n}(v)$, and any two different vertices at distance 2 from $v$ are adjacent to different 4 -sets of vertices in $\Gamma_{n}(v)$, the vertices at distance 2 from $v$ are all fixed by $H$. In particular $\Gamma_{n}(u)$ is fixed by $H$ for any $u \in \Gamma_{n}(v)$. Hence all the vertices at distance 2 from $u$ are fixed, too, and $H=1$, as claimed. This completes the proof for $n>6$.

The graph $\Gamma_{6}$ is a double antipodal cover of $K_{10}$, and $\operatorname{Aut}\left(\Gamma_{6}\right) \cong 2 \times \operatorname{Sym}(6)$. We show that nevertheless only $\operatorname{Sym}(6)$ arises as $\operatorname{Aut}\left(\bar{G}_{6}\right)$. Indeed, the normal subgroup $H=\operatorname{Sym}(2)$ interchanges simultaneously all the vertices of $\Gamma_{6}$ at the maximal distance; they correspond to Triangles of $\bar{G}_{6}$ with no common nonzero coordinate. On the other hand, $H$ must act on $\bar{G}_{6}$. Observe that the pointwise stabilizer in $\operatorname{Sym}(6)$ of the facet $t_{1}=\left(x_{12},-x_{13},-x_{23}\right)$, that belongs to a Triangle $T_{1}$, acts transitively on the three facets with coordinates $x_{45}, x_{46}$ and $x_{56}$ forming a Triangle $T_{2}$. On the other hand $H$ must map $t_{1}$ to one of these three latter facets, as it must interchange $T_{1}$ and $T_{2}$. This means that $H$ does not commute with the action of $\operatorname{Sym}(6)$ on the vertices of $\bar{G}_{6}$, contradicting the assumption that $H \times \operatorname{Sym}(6)$ acts there. This completes the proof in the remaining case $n=6$.

\subsubsection{Is $\left(\mathrm{Met}_{4}\right)$}

Here we shall construct the group of isometries of Met $_{4}$ as the reflection group (see e.g. [11]) $A_{2} \times A_{3} \cong \operatorname{Sym}(3) \times \operatorname{Sym}(4)$. Let us recall the definition of a reflection $s(\alpha)$ with respect to the hyperplane orthogonal to the vector $0 \neq \alpha \in V$, with $V$ the Euclidean with the scalar product $\langle$,$\rangle .$

$$
s(\alpha) v=v-2 \frac{\langle v, \alpha\rangle}{\langle\alpha, \alpha\rangle} \alpha, \quad v \in V .
$$

Since $\mathrm{Met}_{4}=\mathrm{Cut}_{4}$, the 7 extreme rays of $\mathrm{Met}_{4}$ are just the 7 nonzero cuts of the graph $K_{4}$. In other words, a cut $V_{1} \cup V_{2}$ of $K_{4}$, i.e., a partition of the vertices of $K_{4}$ into two parts $V_{1}$ and $V_{2}$, corresponds to the semimetric $d$ satisfying $d(x, y)=1$ for $x$ and $y$ from different parts of the cut, and $d(x, y)=0$ otherwise. Writing the above-the-main-diagonal contents 
of $4 \times 4$ symmetric matrices as vectors, the cuts $r_{1}, \ldots, r_{7}$ are as follows:

$$
\begin{array}{rllllll}
r_{1}= & (0 & 0 & 1 & 0 & 1 & 1) \\
r_{2}=(0 & 1 & 1 & 1 & 1 & 0) \\
r_{3}=(0 & 1 & 0 & 1 & 0 & 1) \\
r_{4}=(1 & 0 & 1 & 1 & 0 & 1), \\
r_{5}=(1 & 1 & 0 & 0 & 1 & 1) \\
r_{6}=(1 & 0 & 0 & 1 & 1 & 0) \\
r_{7}=(1 & 1 & 1 & 0 & 0 & 0)
\end{array} \quad\left(r_{4}, r_{5}\right)=s(0,-1,1,1,-1,0)
$$

Under the action of $\operatorname{Sym}(4)$, there are 2 orbits, one of 4 vectors $r_{1}, r_{3}, r_{6}, r_{7}$ with 3 nonzero entries, and the other of the remaining 3 vectors.

We construct 5 reflections that generate the group $\operatorname{Sym}(3) \times \operatorname{Sym}(4)$ acting on set (5) of vectors. Each reflection is determined by the hyperplane, generated by 5 linearly independent elements of (5). More precisely, the reflection $s(\alpha)$ acting as the permutation $\left(r_{i}, r_{j}\right)$ is given by an $\alpha \neq 0$ in the kernel of the $5 \times 6$ matrix $\left(\begin{array}{l}r_{\ell_{1}} \\ r_{\xi_{5}}\end{array}\right)$, where $\ell_{j} \neq i, j$. Note that it is necessary that $r_{i}$ and $r_{j}$ lie in the same orbit of $\operatorname{Sym}(4)$. For instance, $\alpha$ for $\left(r_{4}, r_{5}\right)$ is given in (5).

It is straightforward to check that indeed the 5 reflections just described generate the group $A_{2} \times A_{3}$, and that they act on the rays $r_{i}$ 's in (5). Moreover, it suffices to check that one of these reflections acts on the rays, as together with the already present $\operatorname{Sym}(4)$ they generate the whole group in question.

To complete the proof of Theorem 1 in this case, it suffices to refer to the fact that the ridge graph $G_{4}$ of Met $_{4}$ is isomorphic to the line graph of $K_{3,4}$ (cf. [5]), and thus the symmetry group cannot be bigger than its automorphism group $\operatorname{Sym}(3) \times \operatorname{Sym}(4)$.

\subsection{The group $I s\left(\mathrm{Cut}_{n}\right)$ for $n \geq 4$}

As $\mathrm{Cut}_{4}=\mathrm{Met}_{4}$, we can assume that $n>4$. First, we remind that the maximal size facets of $\mathrm{Cut}_{n}$ are the triangle facets given in (1) and that a pair of triangle facets are adjacent in the ridge graph of $\mathrm{Cut}_{n}$ if and only if they are adjacent in the ridge graph of $\mathrm{Met}_{n}$.

Lemma 1 ([6]). Any facet of $\mathrm{Cut}_{n}$ contains at most $3 \cdot 2^{n-3}-1$ extreme rays (cuts) with equality if and only if it is a triangle facet.

Lemma 2 ([5]). A pair of triangle facets of $\mathrm{Cut}_{n}$ intersect on a face of codimension 2 if and only if they are non-conflicting.

Lemmas 1 and 2 imply that the ridge graph of $\mathrm{Met}_{n}$ is an induced subgraph in the ridge graph of Cut that is invariant under any isometry of $\mathrm{Cut}_{n}$. Therefore we have $I s\left(\mathrm{Cut}_{n}\right)=I s\left(\mathrm{Met}_{n}\right)$.

\subsection{The group $I s\left(C_{n}\right)$ for $\mathrm{Cut}_{n} \subseteq C_{n} \subseteq \operatorname{Met}_{n}$}

Let $C_{n}$ be a cone having, among others, the cuts as extreme rays and for which, among others, the triangle inequalities as facet-inducing. As $\mathrm{Cut}_{4}=\mathrm{Met}_{4}$, we can assume that $n>4$. In the same way as for Lemma 1, Lemma 3 can be directly deduced from a similar statement for the cut polytope $\mathrm{cut}_{n}$, see [4] and also [9, Proposition 26.3.12]. 
Lemma 3. Let $C_{n}$ be a cone satisfying $\mathrm{Cut}_{n} \subseteq C_{n} \subseteq \mathrm{Met}_{n}$, any facet of $C_{n}$ contains at most $3 \cdot 2^{n-3}-1$ cuts with equality if and only if it is a triangle facet.

Since $\mathrm{Cut}_{n} \subseteq C_{n} \subseteq \mathrm{Met}_{n}$ and triangle facets are adjacent in the ridge graphs of both $\mathrm{Cut}_{n}$ and $\mathrm{Met}_{n}$ if and only if they are non-conflicting, we have the following.

Lemma 4. Let $C_{n}$ be a cone satisfying $\mathrm{Cut}_{n} \subseteq C_{n} \subseteq$ Met $_{n}$, a pair of triangle facets of $C_{n}$ intersect on a face of codimension 2 if and only if they are non-conflicting.

As in Section 3.2, Lemmas 3 and 4 imply that the ridge graph of Met ${ }_{n}$ is an induced subgraph in the ridge graph of $C_{n}$ that is invariant under any isometry of $C_{n}$. This completes the proof of Theorem 2.

Acknowledgments The authors thank Mikhail Klin and Monique Laurent for useful remarks and supplying relevant references.

The first author is partially supported by the NSREC under the Canada Research Chair and the Discovery Grant programs.

The work was completed while the third author held a position at CS Dept. of University of Frankfurt, supported by the DFG Grant SCHN-503/2-1. The second and the third author acknowledge support by the Research School SOM of the University of Groningen.

\section{References}

1. P. Assouad and M. Deza, Metric subspaces of $l^{1}$. Publications mathématiques d'orsay 82-03, Université de Paris Sud, Orsay, 1982.

2. A.E. Brouwer, A.M. Cohen, and A. Neumaier, Distance-Regular Graphs, Springer-Verlag, BerlinHeidelberg-New York, 1989.

3. P.J. Cameron, Permutation Groups, volume 45 of London Mathematical Society Student Texts, Cambridge University Press, Cambridge, 1999.

4. A. Deza and M. Deza, "On the skeleton of the dual cut polytope," in Jerusalem combinatorics '93, volume 178 of Contemp. Math., Amer. Math. Soc., Providence, RI, 1994 pp. 101-111.

5. A. Deza and M. Deza, "The ridge graph of the metric polytope and some relatives," in Polytopes: Abstract, convex and computational (Scarborough, ON, 1993), volume 440 of NATO Adv. Sci. Inst. Ser. C Math. Phys. Sci., pp. 359-372. Kluwer Acad. Publ., Dordrecht, 1994.

6. A. Deza, M. Deza, and K. Fukuda, "On skeletons, diameters and volumes of metric polyhedra," in Combinatorics and Computer Science (Brest, 1995), volume 1120 of Lecture Notes in Comput. Sci., pp. 112-128. Springer, Berlin, 1996.

7. M. Deza and M. Dutour, "Data mining for cones of metrics, quasi-metrics, hemi-metrics and supermetrics," e-print, ENS Paris, http://arxiv.org/abs/math.MG/0201011, 2002.

8. M. Deza, V.P. Grishukhin, and M. Laurent, "The symmetries of the cut polytope and of some relatives," in Applied geometry and discrete mathematics, volume 4 of DIMACS Ser. Discrete Math. Theoret. Comput. Sci., pp. 205-220. Amer. Math. Soc., Providence, RI, 1991.

9. M. Deza and M. Laurent, Geometry of Cuts and Metrics, volume 15 of Algorithms and Combinatorics. Springer-Verlag, Berlin, 1997.

10. I.A. Faradžev, M.H. Klin, and M.E. Muzichuk, "Cellular rings and groups of automorphisms of graphs," in Investigations in Algebraic Theory of Combinatorial Objects, volume 84 of Math. Appl. (Soviet Ser.), pp. 1-152. Kluwer Acad. Publ., Dordrecht, 1994.

11. J.E. Humphreys, Reflection groups and Coxeter groups, volume 29 of Cambridge Studies in Advanced Mathematics, Cambridge University Press, Cambridge, 1990.

12. M.C. Klin, R.Pöschel, and K. Rosenbaum, Angewandte Algebra für Mathematiker und Informatiker. VEB Deutscher Verlag der Wissenschaften, Berlin, 1988. Einführung in gruppentheoretisch-kombinatorische Methoden. [Introduction to group-theoretical combinatorial methods].

13. M.H. Klin, A study of algebras of invariant relations of certain classes of permutation groups, $\mathrm{PhD}$ thesis, NKI and Kiev State University, 1974.

14. M. Laurent, "Graphic vertices of the metric polytope," Discrete Math. 151(1-3) (1996),131-153,. Graph theory and combinatorics (Manila, 1991). 